\title{
Editorial
}

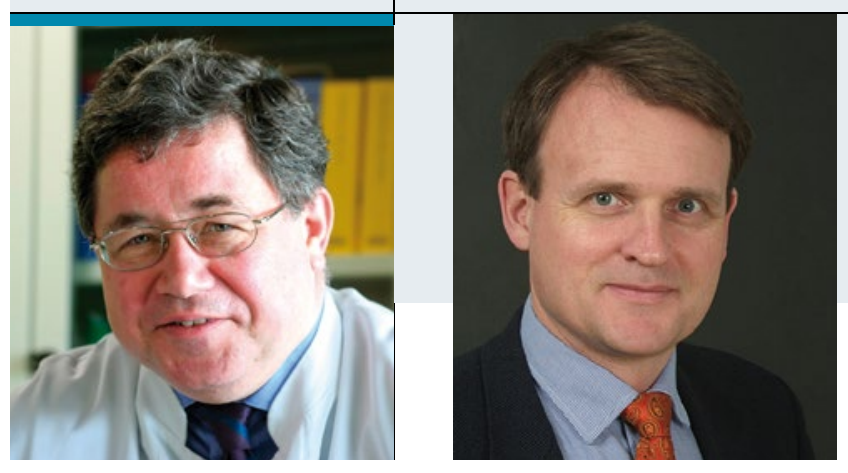

"Das Allergo Journal hat die Entwick-

lungen der molekularen Allergologie in

den vergangenen Jahren mit speziellen

Serien und Übersichten begleitet."

\section{Möglichkeiten der molekularen Allergologie und mehr}

Prof. Dr. Hans F. Merk, Hautklinik der Medizinischen Fakultät, Universitätsklinikum der RWTH Aachen

Prof. Dr. Thilo Jakob, Klinik für Dermatologie und Allergologie, Universitätsklinikum Gießen und Marburg, Standort Gießen

W enngleich die Entwicklung neuer Medikamente heute ganz im Zeichen von Arzneimitteln stehen, die meist zur Gruppe der Peptide oder Proteine gehören und mit spezifischen Targetstrukturen interagieren, besitzen klassische, kleinmolekulare Wirkstoffe immer wieder überraschende Effekte, die neu entdeckt werden. Ein faszinierendes Beispiel hierfür sind Antacida, die durch die Beeinflussung der Magen-Darm-Passage die Entstehung von Allergien auf Nahrungsmittel, aber auch auf Medikamente begünstigen können. Die spannenden Experimente, die zur Erkennung dieses Zusammenhangs führten, werden in dieser Ausgabe von Eva Untersmayr dargestellt (S. 25), die das Verdienst hat, diese Wechselwirkung zuerst beobachtet zu haben und sie in mehreren Studien belegen konnte.

Das Allergo Journal hat die Entwicklungen der molekularen Allergologie in den vergangenen Jahren mit speziellen Serien und Übersichten begleitet - so wird auch in dieser Ausgabe die Serie „Molekulare Allergologie" mit zwei Beiträgen fortgesetzt.

Will man die Möglichkeiten der molekularen Allergologie in der Diagnostik nutzen und ausschöpfen, sind auch neue technische Plattformen notwendig, die im CME-Beitrag „Molekulare Allergiediagnostik im Multiplex-Verfahren" auf S. 42 ausführlich dargestellt werden. Die zweite Übersichtsarbeit zur molekularen Diagnostik bezieht sich speziell auf die Milch- und Hühnereiweißsensibilisierung und zeigt den Nutzen, aber auch die Grenzen dieser Methode auf, um prognostische Aussagen für die Patienten zu treffen (S. 34). Hilfreich gerade bei der Milch- und Hühnereiweißallergie ist übrigens die klinische Vereinfachung der Probenentnahme bei Kindern [Ott $\mathrm{H}$ et al. Clinical usefulness of microarray-based IgE detection in children with suspected food allergy. Allergy. 2008;63:1521-8 / Ott H et al. Microarray-based IgE detection in capillary blood samples of patients with atopy. Allergy 2006;61:1146-7].

In der bereits erwähnten Übersicht zu MultiplexTechnologien in der Allergiediagnostik wird auch auf die möglichen Auswirkungen für die spezifische Immuntherapie hingewiesen, bei der die gewünschte Wirkung bei vertretbaren unerwünschten Effekten (vgl. Originalarbeit von Distler et al., S. 16) optimiert werden soll.

In der Bewertung von Präparaten für die spezifische Immuntherapie schleicht sich immer wieder der aus der Leitliniendiskussion stammende Begriff der Evidenz ein. Hier sollte bedacht werden, dass eine geringe Wirksamkeit eine hohe Evidenz und eine starke Wirkung geringe Evidenz haben kann und daher in der Beurteilung eines Präparats der Wirksamkeitsnachweis im Zentrum stehen sollte. Dabei kann berücksichtigt werden, dass gegenwärtig verfügbare Präparate Charakteristika von Biosimilars besitzen, die aus der aktuellen Diskussion um Biologics bekannt sind. Diese Aspekte sollten bei aktuellen Diskussionen um die Stellung einzelner Präparate zur spezifischen Immuntherapie bedacht werden, wie auch in der Stellungnahme des Ärzteverbands Deutscher Allergologen (AeDA), der Deutschen Gesellschaft für Allergologie und klinische Immunologie (DGAKI) und der Gesellschaft für Pädiatrische Allergologie und Umweltmedizin (GPA) in diesem Heft dargelegt wird (S. 68).

Mit diesen spannenden Themen rund um die aktuelle Allergologie wünschen wir Ihnen ein frohes Weihnachtsfest und ein gutes neues Jahr.

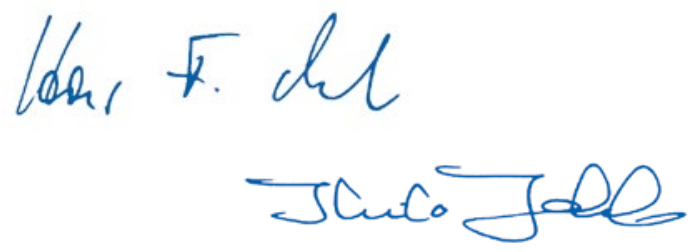

